\title{
Integrin-mediated internalization of Staphylococcus aureus does not require vinculin
}

\author{
Marina Borisova', Yong Shi ${ }^{1}$, Alexander Buntru ${ }^{1,2}$, Susanne Wörner ${ }^{1}$, Wolfgang H Ziegler ${ }^{3}$ and Christof R Hauck ${ }^{1,2^{*}}$
}

\begin{abstract}
Background: Disease manifestations of Staphylococcus aureus are connected to the fibronectin (Fn)-binding capacity of these Gram-positive pathogens. Fn deposition on the surface of $S$. aureus allows engagement of a5 $\beta 1$ integrins and triggers uptake by host cells. For several integrin- and actin-associated cytoplasmic proteins, including FAK, Src, N-WASP, tensin and cortactin, a functional role during bacterial invasion has been demonstrated. As reorganization of the actin cytoskeleton is critical for bacterial entry, we investigated whether vinculin, an essential protein linking integrins with the actin cytoskeleton, may contribute to the integrin-mediated internalization of $S$. aureus.

Results: Complementation of vinculin in vinculin -/- cells, vinculin overexpression, as well as shRNA-mediated vinculin knock-down in different eukaryotic cell types demonstrate, that vinculin does not have a functional role during the integrin-mediated uptake of $S$. aureus.

Conclusions: Our results suggest that vinculin is insignificant for the integrin-mediated uptake of S. aureus despite the critical role of vinculin as a linker between integrins and F-actin.
\end{abstract}

Keywords: Staphylococcus aureus, Bacterial adhesion, Endocytosis, Fibronectin, Host cell invasion, Integrin, Vinculin

\section{Background}

Staphylococcus aureus is often associated with nosocomial infections, which can lead to life threatening conditions such as acute endocarditis and bacteremia. Pathogenesis by $S$. aureus involves several bacterial virulence factors including exotoxins and adhesins [1]. Six different adhesins of S. aureus (FnBPA, FnBPB, Eap, Emb, Ebh and Aaa/Sle1) were found to bind to the extracellular matrix (ECM) protein fibronectin [2]. In particular, bacterial cell wall anchored fibronectin binding proteins (FnBPs) can capture soluble fibronectin from host plasma [3]. Binding to fibronectin is mediated by several repeats within FnBP, which interact with the amino-terminal fibronectin type I domains of this host glycoprotein [4]. Via an RGD motif within one of the fibronectin type III domains, S. aureusassociated fibronectin is able to bind with high affinity to integrin $\alpha 5 \beta 1$. Accordingly, fibronectin can serve as a

\footnotetext{
* Correspondence: christof.hauck@uni-konstanz.de

'Lehrstuhl Zellbiologie, Universität Konstanz, Postfach X908, 78457 Konstanz, Germany

${ }^{2}$ Konstanz Research School Chemical Biology, Universität Konstanz, 78457

Konstanz, Germany

Full list of author information is available at the end of the article
}

molecular bridge connecting $S$. aureus with host cell receptors $[5,6]$. Moreover, the FnBP-fibronectin mediated engagement of integrins triggers internalization of the microbes by non-professional phagocytes such as epithelial cells, endothelial cells, keratinocytes, and fibroblasts [7-10]. Several investigations demonstrate the importance of the FnBP-mediated invasion process in vivo. In a rat model of endocarditis, $S$. aureus strains with reduced fibronectin-binding capacity showed a decreased ability to colonize damaged heart valves [11]. In addition, FnBP expression enhances the capacity of $S$. aureus to colonize mammary glands and invade mammary epithelial cells in a mouse model of mastitis [12]. Exogenous expression of S. aureus FnBP in non-pathogenic Lactobacillus lactis allows these bacteria to colonize damaged heart valves and to spread to the spleen in a mouse model of endocarditis [13]. Therefore, FnBP-mediated host cell contact and cellular invasion appear to contribute to $S$. aureus survival and persistence within the infected host [14]. As FnBPrelated proteins are found in other human pathogens and as integrin-mediated host cell internalization appears critical for certain manifestations of $S$. aureus infections, a 
better understanding of the molecular mechanisms guiding FnBP-initiated uptake is warranted.

We and others have previously shown that fibronectin deposition on the surface of $S$. aureus allows engagement of $\alpha 5 \beta 1$ integrins and triggers the recruitment of actin- and focal adhesion-associated proteins such as paxillin, zyxin, tensin, cortactin, N-WASp, Arp2/3, and FAK to the sites of bacterial attachment $[5,15,16]$. For several of these proteins, including N-WASP, tensin, FAK and cortactin, a functional role during integrinmediated uptake of $S$. aureus has been demonstrated $[15,16]$. As reorganization of the actin cytoskeleton is crucial for the internalization process $[9,10,17]$, it is assumed that dynamic regulation of F-actin by these proteins contributes to bacterial uptake.

Vinculin is one of the characteristic actin-binding proteins recruited to integrin-rich focal adhesion sites, which mechanically links integrin cytoplasmic tails with the actin cytoskeleton $[18,19]$. Vinculin has no enzymatic activity and its functions are regulated by a conformational switch between a closed (inactive) conformation, mediated by an intramolecular head-tail interaction, and an open (active) state [20]. In the open conformation, the vinculin head and tail domains dissociate, allowing multiple interactions with additional proteins or phospholipids [21]. For example, talin, $\alpha$-actinin, VASP, paxillin, phosphatidylinositol-4,5-bisphosphate, and F-actin bind to active vinculin [18]. In addition, vinculin may promote actin filament nucleation by recruiting the Arp2/3 complex to integrin tails [22]. Furthermore, depending on the conformational state, vinculin can also act as an F-actin barbed end capping protein [23]. A role for vinculin during bacterial entry has been reported in the case of Shigella flexneri. Upon contact with epithelial cells, S. flexneri injects the IpaA protein into the host cell cytoplasm, where IpaA directly binds to vinculin inducing a dramatic rearrangement of the actin cytoskeleton to promote bacterial engulfment $[24,25]$. Vinculin has also been observed to be recruited to $S$. aureus-host cell contact sites or fibronectin-coated beads in epithelial, but not in endothelial cells $[15,16,22]$. However, it has not been investigated if vinculin has a functional role during integrin-mediated internalization of $S$. aureus into host cells.

In this report, we analyze the contribution of vinculin to FnBP-mediated uptake of $S$. aureus in different human and murine cell types. Surprisingly, re-expression of vinculin in vinculin-deficient fibroblasts as well as shRNA-mediated knock-down of this protein in different cell types do not affect bacterial uptake demonstrating that vinculin is completely dispensable for bacterial internalization via integrin $\alpha 5 \beta 1$. Therefore, our results suggest that actin cytoskeleton rearrangements during integrinmediated endocytosis do not require vinculin function despite the well characterized role of vinculin as a linker between integrins and F-actin.

\section{Results}

Enhanced cell invasion of S. aureus into vinculin knockout fibroblasts correlates with increased integrin a5 surface expression

Murine embryonic fibroblasts with a genetic deletion of vinculin (vinculin -/- cells) have been generated and been used in a previous study [26]. When grown on a fibronectin-coated surface, vinculin -/- cells spread more slowly and form smaller, less stable focal adhesions compared to wildtype fibroblasts (vinculin WT cells) or vinculin -/- cells re-expressing vinculin [27,28]. Total internal reflection fluorescence (TIRF) microscopy demonstrates that vinculin is located in well-organized, peripheral focal adhesions in vinculin WT cells. In vinculin -/- cells, no staining with the monoclonal $\alpha$-vinculin antibody was observed (Figure 1A). The absence of vinculin was accompanied by a reduced size and an altered distribution of integrin $\beta 1$-containing focal adhesions at the cell-substrate interface (Figure 1A). Compared to vinculin -/- cells, vinculin WT cells showed concentration of large integrin $\beta 1$-containing focal adhesions in the cell periphery (Figure 1A). However, the overall amount of active integrin $\beta 1$ did not differ between vinculin WT and vinculin -/- cells as detected by a conformation-specific monoclonal antibody (Additional file 1: Figure S1). The lack of vinculin in whole cell lysates (WCLs) prepared from vinculin -/- cells was also confirmed by Western blotting (Figure 1B). Importantly, expression of several known focal adhesion proteins and vinculin binding partners such as talin, FAK, paxillin, and Src was not altered by the absence of vinculin (Figure 1B). Equal loading of cell lysates for Western blotting was confirmed by probing for $\beta$-tubulin (Figure 1B). To test for a functional role of vinculin in the integrin-mediated internalization of bacteria, we infected these fibroblasts with pathogenic S. aureus strain Cowan, which connects to the host cell receptor integrin $\alpha 5 \beta 1$ by binding to the integrin ligand fibronectin. As a negative control, we used nonpathogenic $S$. carnosus, which lacks a fibronectin binding adhesin and which does not invade host cells [5,15]. As expected, host cell adhesion and invasion assays confirmed that $S$. carnosus was unable to invade fibroblasts, whereas $S$. aureus attached to and invaded vinculin WT cells (Figure $1 C$ and D). Unexpectedly, vinculin -/- cells showed significantly increased numbers of cell-associated S. aureus (Figure 1C) and about $40 \%$ increase in host cell invasion (Figure 1D). As vinculin -/- cells have undergone several rounds of selection during their establishment [28], compensatory mechanisms might have occurred in the absence of vinculin. In particular, vinculin -/cells supported increased cell-association of $S$. aureus 


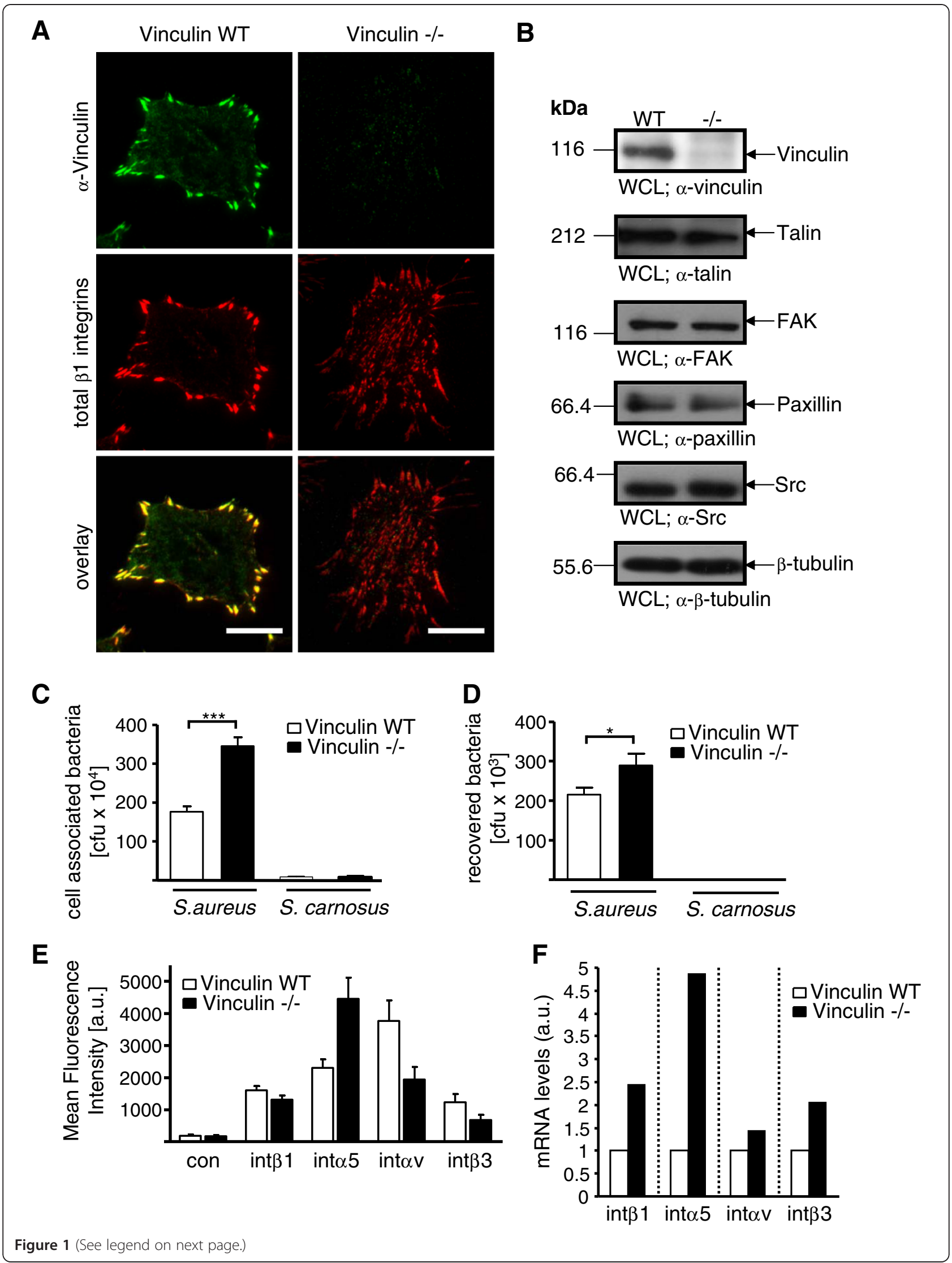


(See figure on previous page.)

Figure 1 Increased integrin a5 expression correlates with enhanced bacterial adherence and uptake in vinculin -/- cells. (A) Vinculin WT and vinculin -/- cells were seeded on fibronectin coated glass bottom imaging dishes, next day fixed and stained for vinculin expression using a-human vinculin antibody (clone hVIN1). Total amounts of integrins were stained using integrin $\beta 1$ antibody (clone HM $\beta 1-1)$. TIRF microscopy was used to assess the localization of vinculin and $\beta 1$ integrins. Bars represent $10 \mu \mathrm{m}$. (B) Equal amounts of protein in the whole cell lysates (WCL) of vinculin WT and vinculin -/- cells were separated by SDS-PAGE and processed for Western blotting using antibodies against talin, FAK, vinculin, paxillin, Src, or $\beta$-tubulin as indicated. Representative blots are shown. (C and D) Cells were seeded on gelatine coated 24 well plates and infected with S. aureus or S. carnosus with $\mathrm{MOI} 20$ for $2 \mathrm{~h}$. Total cell associated or recovered viable intracellular bacteria were determined by bacterial cell adhesion (C) or gentamicin protection assays (D), respectively. Values are means \pm SEM from 5 independent experiments done in quadruplicate $(n=20)$. Statistical significance of data was assessed by Mann-Whitney test. (E) Cells were stained for surface expression of $\beta 1$, a5, $a v$, or $\beta 3$ integrins and examined by flow cytometry. Data are shown as mean fluorescence intensity (MFI) and are corrected for the background MFI of integrin $\beta 1$-/- fibroblasts ( $\beta 1$ integrin staining) or corrected for the MFI of cells stained in the absence of primary antibody (a5, av, and $\beta 3$ integrin stainings). Results are shown as mean \pm SEM from 3 independent experiments. (F) mRNA levels of $\beta 1$, a5, av, or $\beta 3$ integrin transcripts in vinculin WT or vinculin -/- cells were analyzed by qRT-PCR. Integrin expression was normalized to GAPDH expression and was set to 1 for vinculin WT cells. Bars represent the mean of two independent experiments each performed in triplicate.

(Figure 1C), suggesting that the availability of the involved host cell receptor, integrin $\alpha 5 \beta 1$, might be altered in these cells. Therefore, expression of different integrin subunits on the surface of vinculin WT and vinculin -/- cells were investigated by flow cytometry. Whereas integrin $\beta 1$ was present in similar amounts in both cell lines, a strongly increased surface expression of integrin $\alpha 5$ and a reduced expression of the integrin $\alpha v$ subunit was detected in vinculin -/- cells (Figure 1E). Elevated amounts of integrin $\alpha 5$ transcripts were also detected in vinculin -/- cells by quantitative real-time PCR (Figure 1F). The altered integrin $\alpha 5$ expression in vinculin $-/$ - cells correlated with the enhanced host cell binding of S. aureus. Together, these results suggested that the altered integrin expression profile of vinculin WT compared to vinculin -/- cells, and not the absence of vinculin, might be responsible for the observed differences in host cell invasion by $S$. aureus in these murine fibroblast lines.

\section{Re-expression of vinculin in vinculin -/- fibroblasts does not affect bacterial entry}

To address the contribution of vinculin for host cell invasion of $S$. aureus in an identical cellular context, we transiently transfected vinculin -/- cells with a construct encoding enhanced green fluorescent protein (GFP)-tagged murine vinculin. As a control, an GFP encoding vector was used. $48 \mathrm{~h}$ post-transfection, cells seeded on gelatine-coated coverslips were infected with $S$. aureus (MOI 50) for $2 \mathrm{~h}$, fixed and, after blocking with $10 \%$ fetal calf serum in PBS, extracellular bacteria were stained by rabbit $\alpha$-staphylococcal serum and goat $\alpha$-rabbit antibody coupled to Cy5. After cell permeabilization, total cell-associated bacteria were stained by applying rabbit $\alpha$-staphylococcal serum and Cy3-coupled goat $\alpha$-rabbit antibody. The double cycle antibody staining according to this established protocol enables the discrimination between intracellular and extracellular bacteria [29,30]. Fluorescence microscopy pictures revealed that a fraction of GFP-vinculin, but not GFP, localized at focal adhesion sites in the transiently transfected vinculin -/cells (Figure 2A). Moreover, vinculin re-expression was accompanied by a morphological change to a more spread phenotype (Figure 2A). Nevertheless, both GFP as well as GFP-vinculin expressing cells harbored intracellular bacteria (Figure 2A). Enumeration of cell-associated extracellular or intracellular bacteria in GFP versus GFP-vinculin transfected cells demonstrated that bacterial binding and uptake into these cells occurred at a similar level (Figure 2B). Thus, the transient re-expression of vinculin in vinculin -/- cells changes the spreading of the cells, without altering bacterial uptake, indicating that vinculin may not contribute to the internalization of $S$. aureus.

\section{Vinculin overexpression does not influence the uptake of} S. aureus in human cells

Our results that vinculin might not be necessary for integrin-mediated uptake by murine cells were surprising, given by the fact that vinculin is recruited to the sites of bacterial entry in human cells and has a role in F-actin organization and focal adhesion turnover $[15,28]$. To analyze weather vinculin is functionally involved in S. aureus invasion into human cells, we transiently transfected 293T cells to express GFP or GFP-vinculin, respectively. Western blotting with vinculin antibodies confirmed that total vinculin levels were augmented $\sim 10$-fold following transfection with GFP-vinculin $(\sim 145 \mathrm{kDa})$ compared to the endogenous levels of vinculin $(\sim 120 \mathrm{kDa})$ in the GFP transfected cells (Figure 3A). Equal loading of proteins from both samples was confirmed by probing for $\beta$ tubulin (Figure 3A). Expression levels of integrin $\alpha 5$ were only slightly increased in response to vinculin overexpression (Figure 3B). Transfected cells were also seeded on gelatine-coated wells and infected at MOI 20 for $2 \mathrm{~h}$ with the FnBP-expressing S. aureus or the non-invasive $S$. carnosus. Total cell-associated bacteria as well as the numbers of viable intracellular bacteria were then determined. In agreement with our previous observations, the amounts of total cell-associated (Figure $3 \mathrm{C}$ ), as well as the viable 


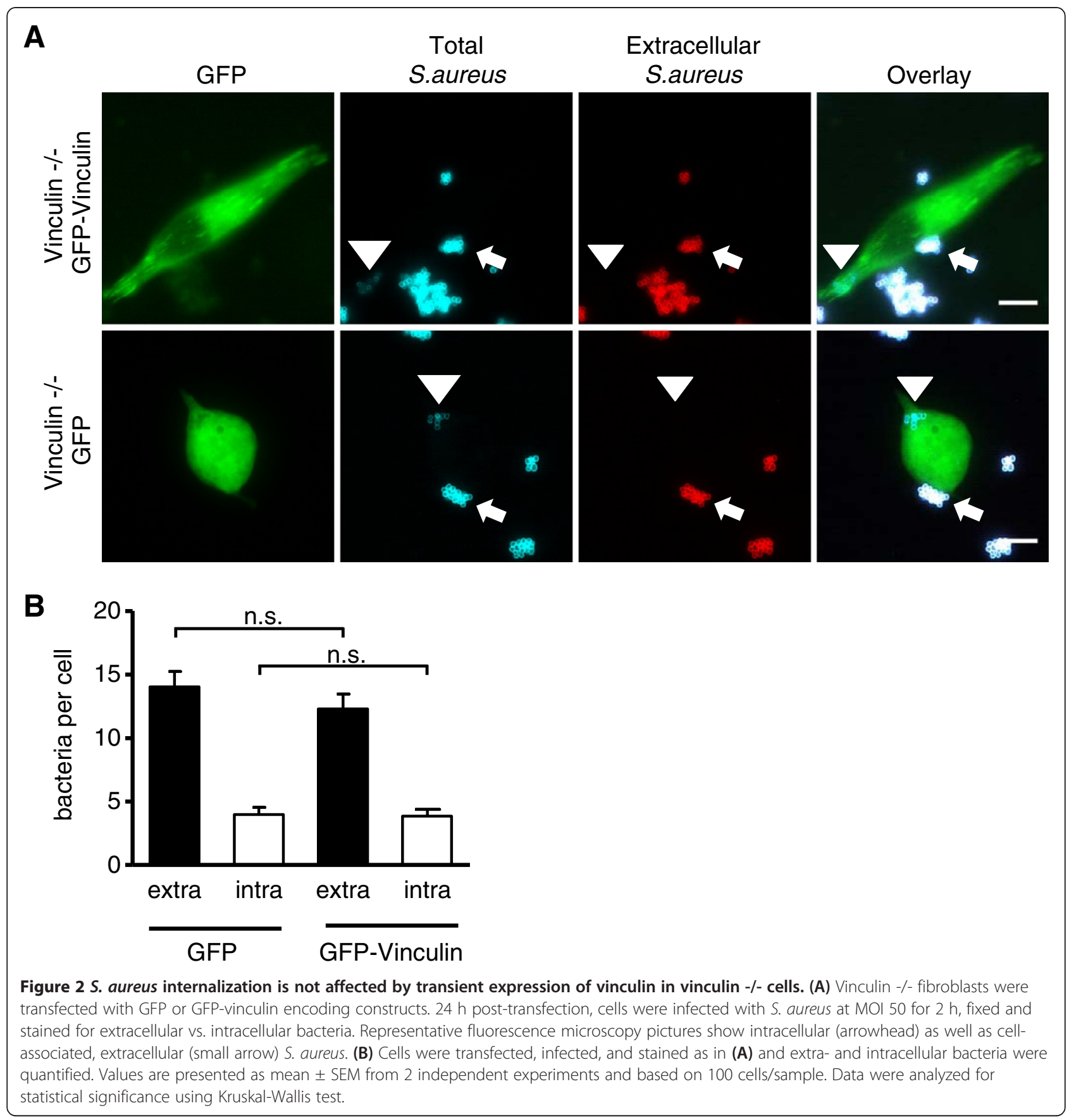

intracellular bacteria were unaltered by vinculin overexpression (Figure 3D). These results demonstrate that vinculin overexpression does not modulate $S$. aureus entry into human cells. These data also indicate that vinculin is not a limiting factor for the integrin-mediated uptake of these fibronectin-binding bacteria and further suggest that the differences observed between murine vinculin WT and vinculin -/ - cells might be due to alterations in the expression pattern of integrin subunits.
ShRNA-mediated vinculin silencing does not interfere with $S$. aureus internalization

Though vinculin is not limiting for S. aureus uptake, endogenous levels of this protein might still be needed to support internalization. Therefore, we investigated whether shRNA-mediated knock-down of vinculin might interfere with $S$. aureus uptake. To this end, we generated murine fibroblasts stably transduced with recombinant lentiviral particles harboring the empty plasmid pLKO.1 
A

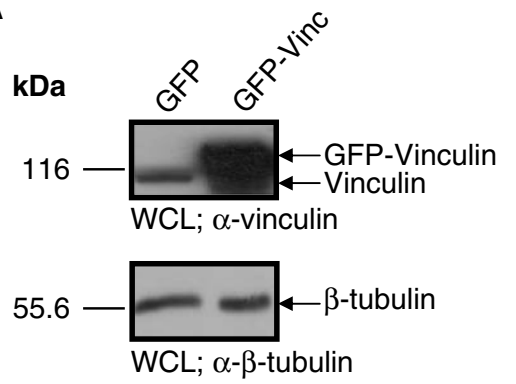

C

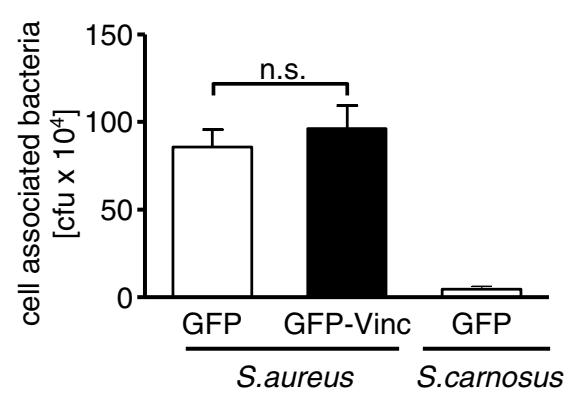

B

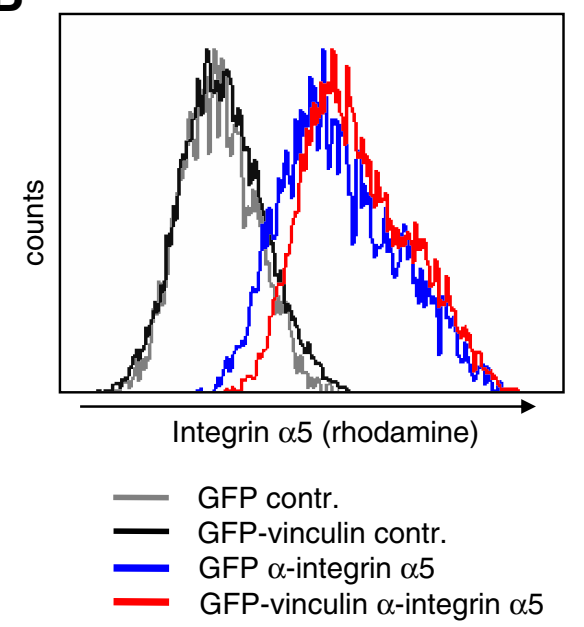

D

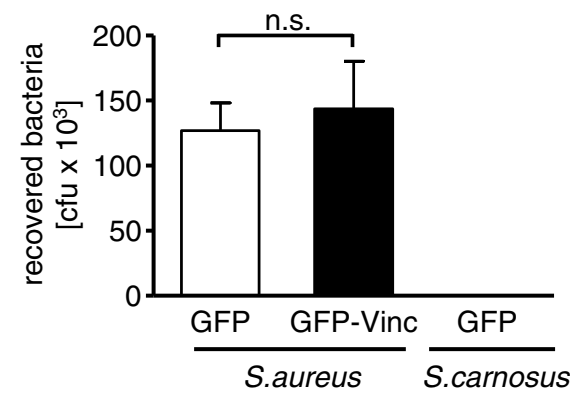

Figure 3 Transient vinculin overexpression in 293 cells does not interfere with bacterial uptake. (A) 293 cells were transfected with constructs encoding GFP or GFP-vinculin. 48 h post-transfection whole cell lysates (WCLs) were prepared and analyzed for vinculin and $\beta$-tubulin expression by immunoblotting. Representative blots are shown. (B) Cells transfected as in (A) were compared for integrin a5 surface expression by flow cytometry. Shown is a representative histogram of cells stained with a-integrin a5 antibody and a rhodamine-coupled secondary antibody (blue - GFP; red - GFP-vinculin) or cells stained with the secondary antibody only (grey lines). (C and D) Cells as in (A) were infected with S. aureus and S. carnosus for $2 \mathrm{~h}$ ( $\mathrm{MOI} 20$ ). Total cell associated or recovered viable intracellular bacteria were determined by bacterial cell adhesion (C) or gentamicin protection assays (D), respectively. Values are means \pm SEM from 2 independent experiments done in quadruplicate, $\mathrm{n}=8$. Mann-Whitney test was applied for statistical significance testing.

or the plasmid pLKO.1 encoding shRNA against murine vinculin (pLKO.1 sh-mvinc). After puromycin selection, the successful vinculin knock-down in the transduced cell population was verified by Western blotting. Importantly, complete inhibition of mouse vinculin expression, but unaltered expression of FAK, Src or paxillin was detected (Figure 4A). Furthermore, surface levels of integrin $\alpha 5$ were similar in vinculin-deficient and vinculin-expressing fibroblasts (Figure 4B). Next, we were interested if the vinculin knock-down could affect interaction of the bacteria with host cells with regard to cell attachment and internalization. Accordingly, pLKO.1 and pLKO.1 shmvinc fibroblasts were infected with $S$. aureus and the amount of total cell-associated as well as the number of viable intracellular bacteria was determined (Figure $4 \mathrm{C}$ ). Though the amounts of cell-associated bacteria were decreased in pLKO.1 sh-mvinc fibroblasts compared to control cells, the number of intracellular bacteria in both cell populations was equal. The results from the bacterial adhesion assay correlated with the slightly decreased expression of integrin $\alpha 5$ on the surface of vinculin knock-down cells, compared to control cells (Figure 4B).

In a second parallel approach, we generated 293 cells stably transduced with recombinant lentiviral particles harboring the empty plasmid pLKO.1 or with particles harboring plasmid pLKO.1 encoding shRNA against human vinculin (pLKO.1 sh-hvinc). After puromycin selection, pLKO.1 sh-hvinc cells showed no detectable vinculin expression in whole cell lysates, but no alteration in FAK, Src and paxillin expression (Figure 5A). Functional assays of bacterial interaction with host cells revealed that knock-down of vinculin in these human cells again did not influence their ability to support fibronectin-mediated cell attachment or internalization of S. aureus (Figure 5B and C). 
A
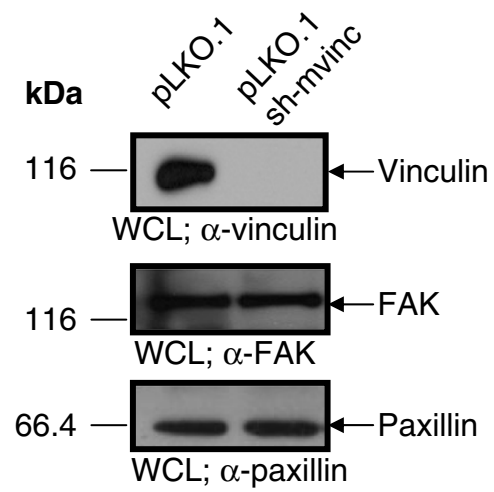

66.4

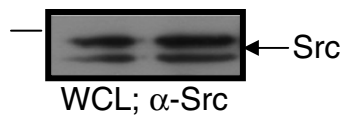

55.6

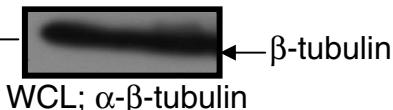

B

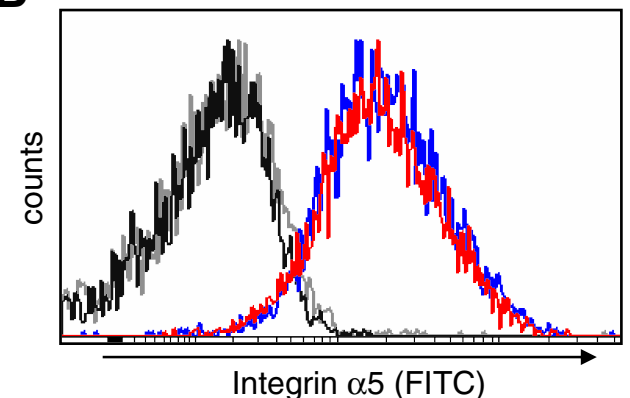

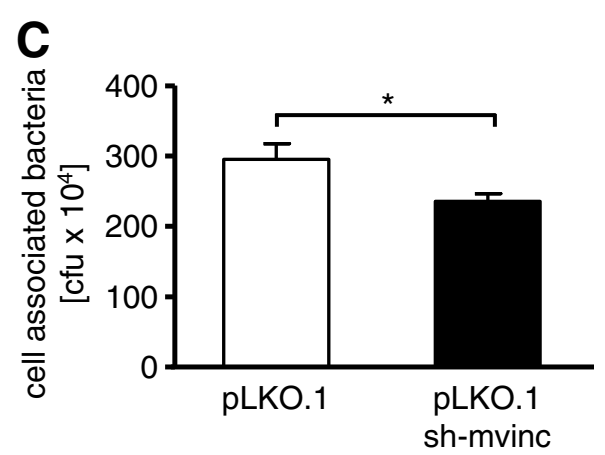

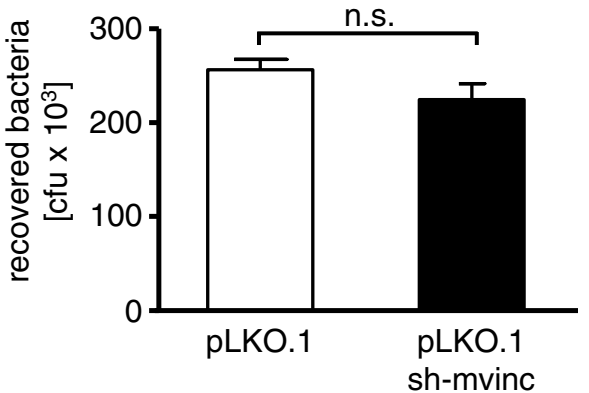

sh-mvinc
- pLKO.1 contr.

- pLKO.1 sh-mvinc contr.

pLKO. $1 \alpha$-integrin $\alpha 5$

pLKO. 1 sh-mvinc $\alpha$-integrin $\alpha 5$

Figure 4 Knock-down of vinculin in wildtype fibroblasts does not affect $\mathbf{S}$. aureus uptake. (A) Murine fibroblasts were transduced with lentiviral particles harboring either pLKO.1 or pLKO.1 encoding shRNA against mouse vinculin (pLKO-1 sh-mvinc). WCL of the stably transduced cell lines were analyzed for FAK, vinculin, paxillin, Src and $\beta$-tubulin protein expression by Western blotting. Representative blots are shown. (B) Integrin a5 surface expression in cells from (A) was analyzed by flow cytometry. Shown is a representative histogram of cells stained with a-integrin a5 antibodies and a rhodamine-coupled secondary antibody (blue - pLKO.1; red - pLKO.1 sh-mvinc) or cells stained with the secondary antibody only (contr.; grey lines). (C) pLKO.1 or pLKO.1 sh-mvinc cells were seeded on gelatine coated 24 well plates. Next day, cells were infected with S. aureus at $\mathrm{MOI} 20$ for $2 \mathrm{~h}$. Total cell associated or recovered viable intracellular bacteria were determined by bacterial cell adhesion (top) or gentamicin protection assays (bottom), respectively. Values are means \pm SEM from 2 independent experiments done in quadruplicate, $\mathrm{n}=8$. Mann-Whitney test was applied for statistical testing.

Taken together, our results demonstrate that vinculin is not required for $S$. aureus invasion in different cell types. Although vinculin might be recruited to the site of bacterial attachment, it has no functional role in orchestrating the integrin-mediated internalization of staphylococci into the eukaryotic host cells.

\section{Discussion}

Since its discovery 32 years ago, vinculin has emerged as a key structural adaptor molecule dynamically linking integrins with the actin cytoskeleton [31]. Here we present experimental evidence that vinculin, despite its reported recruitment, is not required for the integrin-mediated internalization of Staphylococcus aureus by mammalian cells. Our conclusions are based on genetic deletion of vinculin and the complementation of the resulting vinculin-deficient cells, shRNA-mediated knock-down of vinculin, and overexpression of vinculin in different cell types, as all these different treatments do not affect host cell invasion by fibronectin-binding $S$. aureus.

Initially, we were puzzled by the finding that vinculindeficient murine fibroblasts showed slightly elevated association with and uptake of fibronectin-binding S. aureus. However, the immortalized vinculin-deficient embryonic fibroblasts exhibited increased expression of integrin $\alpha 5$ compared to wildtype fibroblasts. It is currently unclear, if the altered expression of integrin $\alpha 5$ is directly caused by the lack of vinculin, which has been reported to associate 

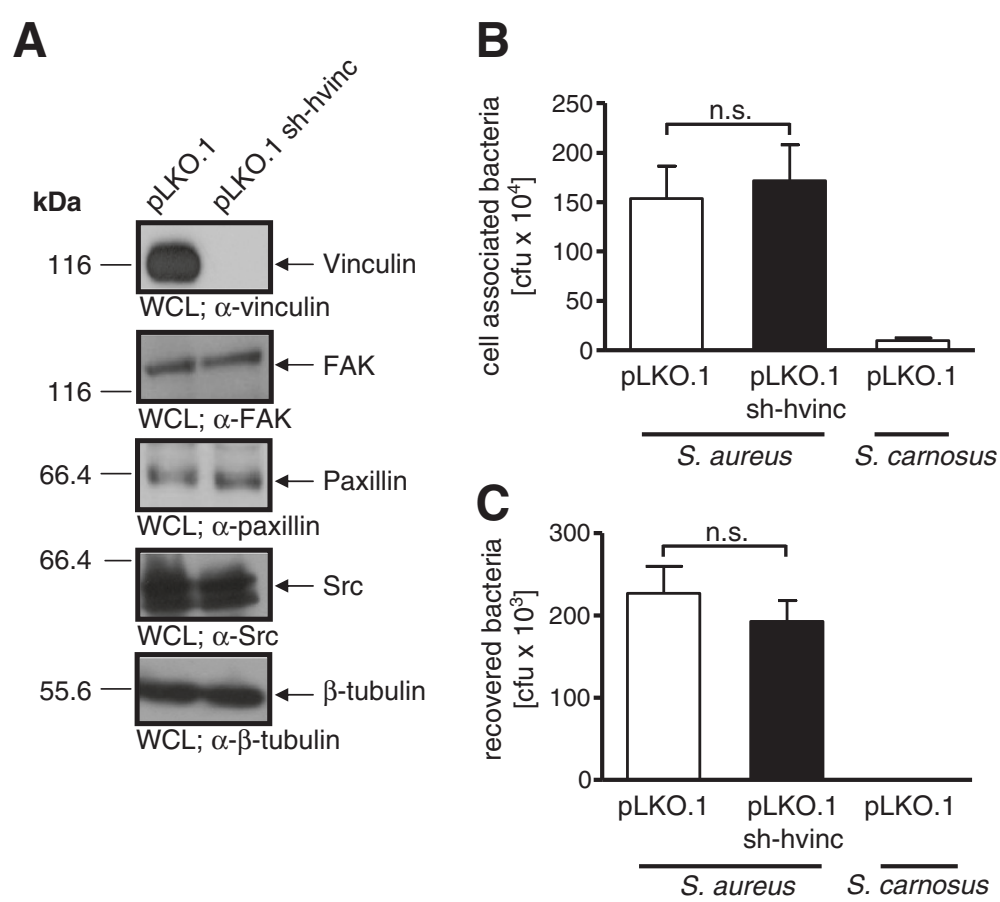

Figure $\mathbf{5}$ Vinculin knock-down in $\mathbf{2 9 3}$ cells does not affect the uptake of S. aureus. (A) 293 cells were stably transduced with pLKO.1 or pLKO.1 encoding shRNA against human vinculin (pLKO.1 sh-hvinc). Whole cell lysates were prepared and WCLs were analyzed by Western blotting for vinculin, FAK, paxillin, SrC, and $\beta$-tubulin expression. Representative blots are shown from 2 independent experiments. (B and C) Cells from (A) were seeded on gelatine coated 24 well plates and infected with S. aureus at MOl 20 for $2 \mathrm{~h}$. Total cell associated or recovered viable intracellular bacteria were determined by bacterial cell adhesion (B) or gentamicin protection assays (C), respectively. Values are means \pm SEM from 4 independent experiments done in triplicate, $n=12$. Data were analyzed for statistical significance using Mann-Whitney test.

with factors involved in mRNA splicing [32], or if this alteration might be due to prolonged in vitro selection and culture of the vinculin-deficient cells. Clearly, re-expression of vinculin in the vinculin-deficient cells did not alter integrin $\alpha 5$ expression and shRNA-mediated knock-down of vinculin in different cell types did not lead to an increase in integrin $\alpha 5$. Therefore, diminishing or increasing vinculin levels in different cell types did neither affect integrin $\alpha 5$ nor integrin-mediated uptake of $S$. aureus suggesting that changes observed in the vinculin -/- cells might have a secondary cause.

Interestingly, vinculin is involved in cell entry of several bacterial pathogens including Shigella flexneri and Bartonella henselae. For example, siRNA screening has revealed that vinculin and other integrin-associated molecules are critical for the formation of the characteristic invasome structure, which mediates entry of the zoonotic pathogen $B$. henselae (Bh) into endothelial cells [33].

In the case of S. flexneri, a leading cause of dysentery worldwide, the entry into human epithelial cells is dramatically impaired in the absence of vinculin [25]. Upon encounter of host cells, S. flexneri injects the IpaA protein. This effector protein contains three vinculin binding sites (VBS), which recognize the $\mathrm{N}$-terminal vinculin head domain $[34,35]$. Therefore, a single IpaA protein may bind up to three vinculin molecules. Importantly, deletion of IpaA or disruption of all three VBS in IpaA severely impair Shigella uptake demonstrating the critical role of vinculin in this entry pathway [35]. Interestingly, the VBS of IpaA are reminiscent of the vinculin binding sites found in the carboxy-terminal rod domain of the focal adhesion protein talin [36]. The eleven VBS of talin also engage the vinculin head domain and help to relief the intramolecular interactions in vinculin [18]. Accordingly, talin or IpaA binding to the vinculin head domain result in exposure of the vinculin tail domain with its binding sites for several proteins including F-actin, $\alpha$-actinin, or the Arp2/3 complex [35,37]. How vinculin in its active conformation promotes Shigella entry is not completely understood, but it has been shown that a vinculin-binding IpaA peptide can modulate the barbed end capping of actin filaments and thereby controls the rate of F-actin polymerization [38]. Similarly, conformational changes in vinculin induced by IpaA may also expose the binding site for the Arp2/3 complex, which is located in a linker region between the vinculin head and tail domain [22]. Again, this would allow Shigella to trigger actin polymerization required for efficient bacterial internalization. 
A further hint, that vinculin can regulate actin polymerization during bacterial entry comes from the study of Helicobacter pylori, an extracellular pathogen, which can engage integrins without being internalized [39]. H. pylori translocates the type IV secretion system effector protein CagA into gastric epithelial cells [40]. Bacterial CagA is initially tyrosine phosphorylated and subsequently inhibits the catalytic activity of Src family protein tyrosine kinases $[41,42]$. The lack of Src activity results in severely reduced phosphorylation of vinculin on residues Y100 and Y1065, disrupting interactions between vinculin and the Arp2/3 complex, and blocking actin cytoskeleton rearrangements in epithelial cells [43]. Together these results suggest that vinculin is a central host factor targeted by several bacterial effector proteins to orchestrate actin cytoskeleton rearrangements to the advantage of the pathogens.

As actin cytoskeleton dynamics are also essential for $S$. aureus host cell entry, yet vinculin does not seem to participate functionally in this process, regulation of actin dynamics might be achieved in a different manner during uptake of fibronectin-binding staphylococci. Previous results indicate that fibronectin-dependent, integrin-mediated internalization of $S$. aureus into host cells requires the integrin-associated tyrosine kinases Src and FAK $[10,15,17]$. One downstream substrate of the active FAK/Src complex is cortactin $[44,45]$. Indeed, cortactin is strongly tyrosine phosphorylated upon contact of fibronectin-binding $S$. aureus with host cells and depletion of cortactin reduces $S$. aureus invasion [15]. Recent studies have demonstrated cortactin directly associates with FAK via SH3 domain-mediated binding to prolinerich sequences in FAK $[45,46]$. As cortactin is also able to organize the F-actin cytoskeleton and to stimulate the actin polymerizing Arp2/3 complex [47,48], recruitment and tyrosine phosphorylation of cortactin might direct the cytoskeletal rearrangements during $S$. aureus internalization, superseding vinculin function downstream of integrin engagement.

Taken together, our investigations come to the surprising conclusion that vinculin is not required for the integrin-mediated uptake of $S$. aureus into host cells. Despite the documented role of vinculin in dynamically linking integrins with the actin cytoskeleton, our results suggest that other cellular factors, such as the FAK-Srccortactin signaling axis, are critical for triggering actin remodeling and bacterial entry during integrin endocytosis. It also appears likely that uptake of other pathogens, which engage integrins for host cell invasion, might be also independent of vinculin function.

\section{Methods}

\section{Cell culture and transfection}

Human embryonic kidney 293T cell line (293 cells, ACC-635, German Collection of Microorganisms and Cell
Cultures, DSMZ, Braunschweig, Germany) was grown in DMEM high glucose (PAA, Pasching, Austria) supplemented with $10 \%$ calf serum (CS). Mouse embryonic fibroblasts were grown on gelatine-coated cell culture dishes in DMEM high glucose supplemented with $10 \%$ fetal calf serum (FCS; Biochrom, Berlin, Germany), non-essential amino acids and sodium pyruvate. Vinculin wildtype and vinculin -/- cells were generated from mice kindly provided by E.D. Adamson (Burnham Institute, La Jolla, CA) [49]. The fibroblasts were newly derived from mouse embryos and immortalized with SV40 largeT antigen as described [26]. All cell cultures were incubated at $37^{\circ} \mathrm{C} / 5 \% \mathrm{CO}_{2}$ and subcultured every 2 to 3 days. Cells were used between passage 2 and 30, counted and assayed for viability with a Casy Cell Counter (Innovatis, Bielefeld, Germany).

Transfection of 293 cells with expression vectors for GFP (Clontech) or GFP-mouse vinculin [50] was accomplished by standard calcium phosphate co-precipitation using $3.5 \mu \mathrm{g}$ of plasmid DNA for each $10 \mathrm{~cm}$ culture dish as previously described [51]. Fibroblasts were transfected using Lipofectamine 2000 reagent (Invitrogen, Carlsbad, CA) according to the manufacturer's instructions. Cells were employed in infection experiments 24 to $48 \mathrm{~h}$ after transfection.

\section{Bacteria}

Staphylococcus aureus Cowan and non-pathogenic $S$. carnosus TM300 have been described before [15]. Bacteria were cultured at $37^{\circ} \mathrm{C}$ and grown on Tryptic Soybean Broth medium (TSB; BD Biosciences, Heidelberg, Germany). Prior to the experiment bacteria were grown to reach a mid-logarithmic growth phase, washed once with PBS and used for infection at multiplicity of infection (MOI) 20 for gentamicin protection assays and MOI 50 for microscopic evaluation of extra- and intracellular bacteria.

\section{shRNA construction and lentiviral production}

Recombinant lentiviral particles were generated using the plasmids pLKO.1, pMD2.G, and psPAX2 provided by Addgene (www.addgene.org) and maintained in $E$. coli STBL4 (Invitrogen, Carlsbad, CA). Using the algorithm $\mathrm{AAGN}_{18} \mathrm{TT}$ (available online at http://jura.wi.mit.edu/ bioc/siRNAext/) sequences that could silence expression of human or mouse vinculin were identified. According to these predictions, complementary primers were synthesized targeting human vinculin mRNA: hVinculin-shRNA-sense $\quad 5^{\prime}$-ccggaaTCAAGCTGCTTATGAA CATctcgagATGTTCATAAGCAGCTTGAttttttg-3' and hVinculin-shRNA-anti $\quad 5^{\prime}$-aattcaaaaaaTCAAGCTGCT TATGAACATctcgagATGTTCATAAGCAGCTTGAtt-3'; or targeting murine vinculin mRNA: mVinculin-shRNAsense 5'-ccggaaATCTGGCTGGTACATACACctcgagGTG 
TATGTACCAGCCAGATtttttttg- $3^{\prime}$ and mVinculinshRNA-anti 5'-aattcaaaaaaaACTGGCTGGTACATACACctcgagGTGTATGTACCAGCCAGATtt-3'. The oligos were annealed and cloned into the AgeI and EcoRI sites of pLKO.1 generating pLKO.1-sh-hvinc and pLKO.1sh-mvinc constructs, respectively. The correct insertion of the shRNA cassette was verified by sequencing.

For lentiviral production, $2 \times 10^{6} 293$ cells were transiently transfected with $14 \mu \mathrm{g}$ of the respective pLKO.1 vector together with $10 \mu \mathrm{g}$ of packaging plasmid psPAX2 and $7 \mu \mathrm{g}$ of envelope-coding plasmid pMD2.G. $48 \mathrm{~h}$ later, the virus-containing cell culture supernatant was collected, centrifuged at $2000 \mathrm{rpm}$ at $4^{\circ} \mathrm{C}$ for $7 \mathrm{~min}$ and filtered through a $0.45 \mu \mathrm{m}$ pore-size filter. $6 \mathrm{ml}$ of the cleared viral supernatant was used to transduce 293 cells or fibroblasts in $10 \mathrm{~cm}$ culture dishes. After $24 \mathrm{~h}$, puromycin $(0.45 \mu \mathrm{g} / \mathrm{ml}$ for 293 cells and $2.5 \mu \mathrm{g} / \mathrm{ml}$ for fibroblasts) was added and the puromycin-resistant stable cell population was used in experiments after 7 days of selection.

\section{Quantification of surface integrin expression by flow cytometry}

Integrin $\alpha 5$ (clone 5H10-27(MFR5)) and integrin $\alpha \mathrm{v}$ (clone RMV-7) antibodies were purchased from BD Biosciences. Integrin $\beta 1$ (clone CBL1333F) was obtained from Cymbus Biotechnology (London, UK) and integrin $\beta 3$ antibody (clone 2C9.G3) from eBioscience (San Diego, CA). Secondary antibodies (biotin-SP-conjugated goat $\alpha$-mouse IgG, biotin-SP-conjugated goat $\alpha$-rat IgG), streptavidin-FITC and streptavidin-rhodamine (TRITC) were purchased from Jackson ImmunoResearch (West Grove, PA). For quantification of surface integrin expression, suspended fibroblasts were incubated in suspension medium (DMEM containing $0.25 \% \mathrm{BSA}$ ) for $40 \mathrm{~min}$ at $37^{\circ} \mathrm{C}$. Then, $2 \times 10^{5}$ cells were incubated with appropriate primary antibodies (diluted 1:500) in FACS buffer (5\% heat-inactivated FCS, $1 \%$ sodium azide in $\mathrm{PBS}$ ) for $1 \mathrm{~h}$ at $4^{\circ} \mathrm{C}$. After washing, secondary antibodies were applied for $1 \mathrm{~h}$ at $4^{\circ} \mathrm{C}$, and after washing, samples were analyzed by flow cytometry (LSRII, BD Biosciences).

\section{qRT-PCR evaluation of integrin mRNA levels}

Total RNA was isolated from vinculin wild type and vinculin -/- cells cells using RNeasy Mini Kit (Qiagen, Hilden, Germany). Reverse transcription was performed on $1 \mu \mathrm{g}$ total RNA. Quantitative real-time PCR was conducted with the sensiMixPlus SYBR Kit (Quantace, Germany) with the following cycle conditions: $95^{\circ} \mathrm{C}$ for $10 \mathrm{~min}$ followed by 40 cycles at $95^{\circ} \mathrm{C}$ for $10 \mathrm{~s}, 60^{\circ} \mathrm{C}$ for $20 \mathrm{~s}$, and $72^{\circ} \mathrm{C}$ for $20 \mathrm{~s}$. Relative expression of $\alpha v_{-}^{-}, \alpha 5-$, $\beta 1-, \beta 3$-integrins was normalized using glyceraldehyde3-phosphate dehydrogenase (GAPDH) according to the method by Livak and Schmittgen [52]. The primers used were: mouse $\alpha v$-integrin (forward: $5^{\prime}$-TTGGG GACGA CAACC CTCTG ACAC-3'; reverse: 5'-TGCGG CGGGA TAGAA ACGAT GAG-3'); mouse $\alpha 5$-integrin (forward: 5'- CACTT GGCTT CAGGG CATTT C-3'; reverse: 5' CAACT ACACC CCCAA CTCAC AGG-3'); mouse $\beta 1$ integrin (forward: 5' - TCTCA CCAAA GTAGA AAGCA GGGA-3'; reverse: $5^{\prime}$ - ACGAT AGCTT CATTG TTGCC ATTC-3'); mouse $\beta 3$-integrin (forward: $5^{\prime}$ - GCTTT GG GGC CTTCG TGGAC AA-3'; reverse: $5^{\prime}$ - CATGG GC AAG CAGGC ATTCT TCAT-3'); and mouse GAPDH (forward: 5' - TGCAC CACCA ACTGCT TAG-3'; reverse: 5'- GGATG CAGGG ATGAT GTTC-3').

\section{Gentamicin protection assay}

Briefly, $2 \times 10^{5} 293$ cells or $1 \times 10^{5}$ fibroblasts were seeded on gelatine coated ( $0.1 \%$ in PBS) 24 well plates. Cells were infected with 20 bacteria per cell (MOI 20) for $2 \mathrm{~h}$. To evaluate the number of intracellular bacteria, the medium was replaced with DMEM containing $50 \mu \mathrm{g} / \mathrm{ml}$ gentamicin. After incubation for $1 \mathrm{~h}$ at $37^{\circ} \mathrm{C}$, intracellular bacteria were released by treatment with $0.5 \%$ saponin in $\mathrm{PBS}$ for $10 \mathrm{~min}$ at $37^{\circ} \mathrm{C}$. Samples were diluted in PBS and plated on TSB agar plates for determination of the recovered colony forming units (cfu). Total cell associated bacteria were determined in separate samples by omitting the incubation with gentamicin and washed once (293) or twice (fibroblasts) with PBS before lysis by $0.5 \%$ saponin in PBS.

\section{Fluorescence staining of vinculin and total internal reflection fluorescence (TIRF) microscopy}

$3 \times 10^{4}$ vinculin WT and vinculin -/- cells were plated on $4 \mu \mathrm{g} / \mathrm{ml}$ fibronectin coated glass bottom imaging dishes. Next day, cells were washed once with $\mathrm{PBS}^{+/+}(\mathrm{PBS}$ supplemented with $\mathrm{Ca}^{2+}$ and $\mathrm{Mg}^{2+}$ ) and fixed with $4 \%$ paraformaldehyde for $20 \mathrm{~min}$. For vinculin staining, fixed samples were washed twice and permeabilized for 10 min in Triton X100 solution (0.5\% in PBS). After three washing steps unspecific binding sites were saturated by incubating the samples with blocking buffer (10\% heat inactivated FCS in $\mathrm{PBS}^{+/+}$) for $10 \mathrm{~min}$. Then, samples were incubated with 1:500 diluted monoclonal mouse $\alpha$-human vinculin antibody (clone hVIN1; SigmaAldrich, Steinheim, Germany) in blocking buffer for $30 \mathrm{~min}$. Samples were washed three times, blocked for $10 \mathrm{~min}$ and incubated with biotin-SP-conjugated goat $\alpha$-mouse IgG antibody (1:500) for $30 \mathrm{~min}$ in blocking buffer. Samples were washed again and incubated for 30 min with streptavidin-FITC (1:500) in the dark. All fluorescence staining steps were performed at room temperature. In addition, samples were co-stained for integrin $\beta 1$ (clone HM $\beta 1-1$; BioLegend, San Diego, CA) together with rhodamine conjugated goat- $\alpha$-armenian hamster IgG antibody (Jackson ImmunoResearch) or for active integrin 
$\beta 1$ (clone 9EG7, recognizing the active conformation of integrin $\beta 1$ ) together with rhodamine conjugated goat- $\alpha$ rat IgG (Jackson ImmunoResearch). Antibodies were diluted 1:300 and samples were analyzed by Total Internal Reflection Fluorescence (TIRF) microscopy. Images were acquired using a Leica AF6000LX TIRF system (Leica, Mannheim, Germany) equipped with 100x/1.46 NA Oil HCX PL Apo objective and EMCCD camera (CascadeII:512). Images were digitally processed using ImageJ.

\section{Cell lysis and western blotting}

Cell lysis and Western blotting were performed as described [51] with some modifications. Briefly, protein concentration was assessed using Pierce bicinchoninic assay kit (Thermo Fisher Scientific, Waltham, MA). Equal amounts of proteins were loaded on SDS-PAGE gels. Antibodies against FAK (clone 77) and paxillin (clone 177) were from BD Biosciences, against talin (clone $\mathrm{H}-300$ ) from Santa Cruz (Santa Cruz, CA) and against vinculin (clone hVIN1) was from Sigma-Aldrich. Antibody against $\beta$-tubulin (E-7) and Src were purified from hybridoma cell supernatants. Goat anti-mouse IgG coupled to HRP was purchased from Jackson ImmunoResearch.

\section{Staining of extra- and intracellular bacteria and fluorescence microscopy evaluation}

$3 \times 10^{4}$ vinculin -/- cells were seeded on gelatine coated acid washed glass cover slips in 24 well plates. Next day, cells were transfected with GFP or GFP-mouse vinculin using Lipofectamine 2000. $24 \mathrm{~h}$ after transfection, cell culture medium was exchanged and cells were infected with Staphylococcus aureus (MOI 50) for 2 h, washed twice with $\mathrm{PBS}^{+/+}$and fixed with $4 \%$ paraformaldehyde in PBS for $20 \mathrm{~min}$ at RT, incubated in blocking buffer for $10 \mathrm{~min}$. Extracellular bacteria were stained using rabbit polyclonal $\alpha$-staphylococcal serum diluted in blocking buffer (45 min at RT). Afterwards, samples were washed three times and incubated with goat $\alpha$-rabbit IgGCy5 (Jackson Immunoresearch) in the dark for $30 \mathrm{~min}$. After three washing steps, cells were permeabilized using $0.5 \%$ Triton/PBS for $10 \mathrm{~min}$, washed three times and blocked additionally for $10 \mathrm{~min}$. Then, after incubation with rabbit polyclonal $\alpha$-staphylococcal serum, samples were washed three times and incubated with goat $\alpha$-rabbit IgG coupled to Cy3 in the dark for $30 \mathrm{~min}$. Finally, after three washes with PBS, the coverslips were mounted in embedding medium (DaKo, Glostrup, Denmark) on glass slides and sealed with nail polish. Images were acquired with a Leica AF6000LX fluorescence microscope and processed with ImageJ.

\section{Statistics}

Infection and flow cytometry experiments were performed two to five times, and data were presented as mean \pm SEM. Differences in adherence and internalization of staphylococci were analyzed by Mann-Whitney test or for more than 2 groups of analysis by Kruskal-Wallis test. In all analyses, $p$ value $<0.05$ was considered statistically significant.

\section{Conclusions}

This study demonstrates that the focal adhesion protein vinculin does not contribute to the integrin-mediated uptake of fibronectin-binding $S$. aureus. This result is unexpected given the critical role of vinculin as a linker between integrins and F-actin.

\section{Additional file}

\begin{abstract}
Additional file 1: Figure S1. Similar distribution of active $\beta 1$ integrins in vinculin -/- and vinculin WT cells. Vinculin WT and vinculin -/- cells were seeded on Fn coated glass bottom imaging dishes, next day fixed and stained for vinculin using mouse a-human vinculin (hVIN1) antibody, combined with biotin-SP-conjugated goat a-mouse lgG and streptavidinFITC. In addition, integrin $\beta 1$ in the ligand-bound, active conformation was detected by rat monoclonal integrin $\beta 1$ antibody (clone 9EG7) together with rhodamine red conjugated goat-a-rat lgG antibody. TIRF microscopy was used to assess the distribution of vinculin and active $\beta 1$ integrins. Bars represent $10 \mu \mathrm{m}$
\end{abstract}

Competing interests

The authors declare that they have no competing interests.

\section{Authors' contributions}

$\mathrm{MB}$ and $\mathrm{CRH}$ conceived the study and designed the experiments, MB and YS performed the experiments, $A B$ advised and performed microscopic determinations, SW generated the vinculin knock down cells, WHZ provided reagents and advised on the manuscript, $M B$ and $C R H$ wrote the paper. All authors read and approved the final manuscript.

\section{Acknowledgments}

We thank NI Dierdorf for designing the shRNAs, C. Hoffmann for preliminary experiments on this project, S. Illenberger for the EGFP-vinculin construct and S. Feindler-Boeckh for the excellent technical support. AB is recipient of a fellowship according to the LGFG, Baden-Württemberg, and acknowledges support by the Zukunftskolleg of the University of Konstanz. This study was supported by funds from the DFG Ha 2856/5-1 (to CRH). The funding body had no influence on experimental design, the collection, analysis, and interpretation of data; the writing of the manuscript; or the decision to submit the manuscript for publication.

\section{Author details}

'Lehrstuhl Zellbiologie, Universität Konstanz, Postfach X908, 78457 Konstanz, Germany. ${ }^{2}$ Konstanz Research School Chemical Biology, Universität Konstanz, 78457 Konstanz, Germany. ${ }^{3}$ Hannover Medical School, Dept. of Paediatric Kidney, Liver and Metabolic Diseases, 30625 Hannover, Germany.

Received: 12 July 2012 Accepted: 21 December 2012 Published: 7 January 2013

\section{References}

1. Lowy FD: Staphylococcus aureus infections. N Engl J Med 1998, 339(8):520-532.

2. Henderson B, Nair S, Pallas J, Williams MA: Fibronectin: a multidomain host adhesin targeted by bacterial fibronectin-binding proteins. FEMS Microbiol Rev 2011, 35(1):147-200.

3. Hauck CR, Ohlsen K: Sticky connections: extracellular matrix protein recognition and integrin-mediated cellular invasion by Staphylococcus aureus. Curr Opin Microbiol 2006, 9(1):5-11. 
4. Schwarz-Linek $U$, Hook $M$, Potts JR: The molecular basis of fibronectinmediated bacterial adherence to host cells. Mol Microbiol 2004, 52(3):631-641

5. Sinha B, Francois PP, Nusse O, Foti M, Hartford OM, Vaudaux P, Foster TJ, Lew DP, Herrmann M, Krause KH: Fibronectin-binding protein acts as Staphylococcus aureus invasin via fibronectin bridging to integrin alpha5beta1. Cell Microbiol 1999, 1(2):101-117.

6. Fowler T, Wann ER, Joh D, Johansson S, Foster TJ, Hook M: Cellular invasion by Staphylococcus aureus involves a fibronectin bridge between the bacterial fibronectin-binding MSCRAMMs and host cell beta1 integrins. Eur J Cell Biol 2000, 79(10):672-679.

7. Dziewanowska K, Patti JM, Deobald CF, Bayles KW, Trumble WR, Bohach GA: Fibronectin binding protein and host cell tyrosine kinase are required for internalization of Staphylococcus aureus by epithelial cells. Infect Immun 1999, 67(9):4673-4678.

8. Massey RC, Kantzanou MN, Fowler T, Day NP, Schofield K, Wann ER, Berendt AR, Hook M, Peacock SJ: Fibronectin-binding protein A of Staphylococcus aureus has multiple, substituting, binding regions that mediate adherence to fibronectin and invasion of endothelial cells. Cell Microbiol 2001, 3(12):839-851.

9. Jett BD, Gilmore MS: Internalization of Staphylococcus aureus by human corneal epithelial cells: role of bacterial fibronectin-binding protein and host cell factors. Infect Immun 2002, 70(8):4697-4700.

10. Agerer F, Michel A, Ohlsen K, Hauck CR: Integrin-mediated invasion of Staphylococcus aureus into human cells requires Src family protein tyrosine kinases. J Biol Chem 2003, 278(43):42524-42531.

11. Kuypers JM, Proctor RA: Reduced adherence to traumatized rat heart valves by a low-fibronectin-binding mutant of Staphylococcus aureus. Infect Immun 1989, 57(8):2306-2312.

12. Brouillette $E$, Talbot BG, Malouin F: The fibronectin-binding proteins of Staphylococcus aureus may promote mammary gland colonization in a lactating mouse model of mastitis. Infect Immun 2003, 71(4):2292-2295.

13. Que YA, Haefliger JA, Piroth L, Francois P, Widmer E, Entenza JM, Sinha B, Herrmann M, Francioli $P$, Vaudaux $P$, et al: Fibrinogen and fibronectin binding cooperate for valve infection and invasion in Staphylococcus aureus experimental endocarditis. J Exp Med 2005, 201(10):1627-1635.

14. Menzies BE: The role of fibronectin binding proteins in the pathogenesis of Staphylococcus aureus infections. Curr Opin Infect Dis 2003, 16(3):225-229.

15. Agerer F, Lux S, Michel A, Rohde M, Ohlsen K, Hauck CR: Cellular invasion by Staphylococcus aureus reveals a functional link between focal adhesion kinase and cortactin in integrin-mediated internalisation. J Cell Sci 2005, 118(10):2189-2200.

16. Schroder A, Schroder B, Roppenser B, Linder S, Sinha B, Fassler R, Aepfelbacher M: Staphylococcus aureus fibronectin binding protein-a induces motile attachment sites and complex actin remodeling in living endothelial Cells. Mol Biol Cell 2006, 17(12):5198-5210.

17. Fowler T, Johansson S, Wary KK, Hook M: Src kinase has a central role in in vitro cellular internalization of Staphylococcus aureus. Cell Microbiol 2003, 5(6):417-426.

18. Ziegler WH, Liddington RC, Critchley DR: The structure and regulation of vinculin. Trends Cell Biol 2006, 16(9):453-460.

19. Geiger B, Spatz JP, Bershadsky AD: Environmental sensing through focal adhesions. Nat Rev Mol Cell Biol 2009, 10(1):21-33.

20. Bakolitsa C, Cohen DM, Bankston LA, Bobkov AA, Cadwell GW, Jennings L, Critchley DR, Craig SW, Liddington RC: Structural basis for vinculin activation at sites of cell adhesion. Nature 2004, 430(6999):583-586

21. Humphries JD, Wang P, Streuli C, Geiger B, Humphries MJ, Ballestrem C: Vinculin controls focal adhesion formation by direct interactions with talin and actin. J Cell Biol 2007, 179(5):1043-1057.

22. DeMali KA, Barlow CA, Burridge K: Recruitment of the Arp2/3 complex to vinculin: coupling membrane protrusion to matrix adhesion. $J$ Cell Biol 2002, 159(5):881-891.

23. Le Clainche C, Dwivedi SP, Didry D, Carlier MF: Vinculin is a dually regulated actin filament barbed end-capping and side-binding protein. J Biol Chem 2010, 285(30):23420-23432.

24. Bourdet-Sicard R, Rudiger M, Jockusch BM, Gounon P, Sansonetti PJ, Nhieu GT: Binding of the Shigella protein IpaA to vinculin induces F-actin depolymerization. EMBO J 1999, 18(21):5853-5862.

25. Tran Van Nhieu G, Ben-Ze'ev A, Sansonetti PJ: Modulation of bacterial entry into epithelial cells by association between vinculin and the Shigella IpaA invasin. EMBO J 1997, 16:2717-2729.
26. Mierke CT, Kollmannsberger P, Zitterbart DP, Diez G, Koch TM, Marg S, Ziegler WH, Goldmann WH, Fabry B: Vinculin facilitates cell invasion into three-dimensional collagen matrices. J Biol Chem 2010, 285(17):13121-13130.

27. Xu W, Coll JL, Adamson ED: Rescue of the mutant phenotype by reexpression of full-length vinculin in null F9 cells; effects on cell locomotion by domain deleted vinculin. J Cell Sci 1998, 111(Pt 11):1535-1544.

28. Saunders RM, Holt MR, Jennings $L$, Sutton DH, Barsukov IL, Bobkov A, Liddington RC, Adamson EA, Dunn GA, Critchley DR: Role of vinculin in regulating focal adhesion turnover. Eur J Cell Biol 2006, 85(6):487-500

29. Heesemann J, Laufs R: Double immunofluorescence microscopic technique for accurate differentiation of extracellularly and intracellularly located bacteria in cell culture. J Clin Microbiol 1985, 22:168-175.

30. Agerer F, Waeckerle S, Hauck CR: Microscopic quantification of bacterial invasion by a novel antibody-independent staining method. J Microbiol Meth 2004, 59(1):23-32

31. Ziegler WH, Gingras AR, Critchley DR, Emsley J: Integrin connections to the cytoskeleton through talin and vinculin. Biochem Soc Trans 2008, 36(Pt 2):235-239.

32. Lahmann I, Fabienke M, Henneberg B, Pabst O, Vauti F, Minge D, Illenberger S, Jockusch BM, Korte M, Arnold HH: The hnRNP and cytoskeletal protein raver1 contributes to synaptic plasticity. Exp Cell Res 2008, 314(5):1048-1060

33. Truttmann MC, Misselwitz B, Huser S, Hardt WD, Critchley DR, Dehio C: Bartonella henselae engages inside-out and outside-in signaling by integrin beta1 and talin1 during invasome-mediated bacterial uptake. J Cell Sci 2011, 124(Pt 21):3591-3602.

34. Izard T, Tran Van Nhieu G, Bois PR: Shigella applies molecular mimicry to subvert vinculin and invade host cells. J Cell Biol 2006, 175(3):465-475.

35. Park H, Valencia-Gallardo C, Sharff A, Tran Van Nhieu G, Izard T: Novel vinculin binding site of the IpaA invasin of Shigella. J Biol Chem 2011, 286(26):23214-23221.

36. Gingras AR, Ziegler WH, Frank R, Barsukov IL, Roberts GC, Critchley DR Emsley J: Mapping and consensus sequence identification for multiple vinculin binding sites within the talin rod. J Biol Chem 2005, 280 (44):37217-37224

37. Hamiaux C, van Eerde A, Parsot C, Broos J, Dijkstra BW: Structural mimicry for vinculin activation by IpaA, a virulence factor of Shigella flexneri. EMBO Rep 2006, 7(8):794-799.

38. Ramarao N, Le Clainche C, Izard T, Bourdet-Sicard R, Ageron E, Sansonetti PJ, Carlier MF, Tran Van Nhieu G: Capping of actin filaments by vinculin activated by the Shigella IpaA carboxyl-terminal domain. FEBS Lett 2007, 581(5):853-857.

39. Kwok T, Zabler D, Urman S, Rohde M, Hartig R, Wessler S, Misselwitz R, Berger J, Sewald N, Konig W, et al: Helicobacter exploits integrin for type IV secretion and kinase activation. Nature 2007, 449(7164):862-866.

40. Backert S, Selbach M: Role of type IV secretion in Helicobacter pylori pathogenesis. Cell Microbiol 2008, 10(8):1573-1581.

41. Selbach M, Moese S, Hauck CR, Meyer TF, Backert S: Src Is the Kinase of the Helicobacter pylori CagA Protein in Vitro and in Vivo. J Biol Chem 2002, 277(9):6775-6778

42. Selbach M, Moese S, Hauck CR, Meyer TF, Backert S: The Helicobacter pylori CagA protein induces cortactin dephosphorylation and actin rearrangements by c-Src inactivation. EMBO J 2003, 22:515-528.

43. Moese S, Selbach M, Brinkmann V, Karlas A, Haimovich B, Backert S, Meyer TF: The Helicobacter pylori CagA protein disrupts matrix adhesion of gastric epithelial cells by dephosphorylation of vinculin. Cell Microbiol 2007, 9(5):1148-1161

44. Wu H, Parsons JT: Cortactin, an 80/85-kilodalton pp60src substrate, is a filamentous actin-binding protein enriched in the cell cortex. J Cell Biol 1993, 120(6):1417-1426.

45. Wang W, Liu Y, Liao K: Tyrosine phosphorylation of cortactin by the FAK-Src complex at focal adhesions regulates cell motility. BMC Cell Biol 2011, 12:49

46. Tegtmeyer N, Wittelsberger R, Hartig R, Wessler S, Martinez-Quiles N, Backert $S$ : Serine phosphorylation of cortactin controls focal adhesion kinase activity and cell scattering induced by Helicobacter pylori. Cell Host Microbe 2011, 9(6):520-531 
47. Weaver AM, Heuser JE, Karginov AV, Lee WL, Parsons JT, Cooper JA: Interaction of cortactin and N-WASp with Arp2/3 complex. Curr Biol 2002, 12(15):1270-1278.

48. Weed SA, Karginov AV, Schafer DA, Weaver AM, Kinley AW, Cooper JA, Parsons JT: Cortactin localization to sites of actin assembly in lamellipodia requires interactions with F-actin and the Arp2/3 complex [In Process Citation]. J Cell Biol 2000, 151(1):29-40.

49. Xu W, Baribault $H$, Adamson ED: Vinculin knockout results in heart and brain defects during embryonic development. Development 1998, 125(2):327-337.

50. Chandrasekar I, Stradal TE, Holt MR, Entschladen F, Jockusch BM, Ziegler WH: Vinculin acts as a sensor in lipid regulation of adhesion-site turnover. J Cell Sci 2005, 118(Pt 7):1461-1472.

51. Schmitter T, Agerer F, Peterson L, Muenzner P, Hauck CR: Granulocyte CEACAM3 is a phagocytic receptor of the innate immune system that mediates recognition and elimination of human-specific pathogens. J Exp Med 2004, 199:35-46.

52. Livak KJ, Schmittgen TD: Analysis of relative gene expression data using real-time quantitative $P C R$ and the $2(-\Delta \Delta C(T))$ Method. Methods 2001, 25(4):402-408.

doi:10.1186/1471-2121-14-2

Cite this article as: Borisova et al:: Integrin-mediated internalization of Staphylococcus aureus does not require vinculin. BMC Cell Biology 2013 14:2.

\section{Submit your next manuscript to BioMed Central and take full advantage of:}

- Convenient online submission

- Thorough peer review

- No space constraints or color figure charges

- Immediate publication on acceptance

- Inclusion in PubMed, CAS, Scopus and Google Scholar

- Research which is freely available for redistribution 